Published in final edited form as:

J Am Chem Soc. 2006 November 29; 128(47): 15108-15110.

\title{
X-ray Crystal Structures of Manganese(II)-Reconstituted and Native Toluene/o-Xylene Monooxygenase Hydroxylase Reveal Rotamer Shifts in Conserved Residues and an Enhanced View of
} the Protein Interior

\author{
Michael S. McCormick, Matthew H. Sazinsky, Karen L. Condon, and Stephen J. Lippard \\ Department of Chemistry, Massachusetts Institute of Technology, Cambridge, Massachusetts \\ 02139 E-mail: lippard@mit.edu
}

Non-heme diiron enzymes perform important functions in biology, ${ }^{1}$ including the selective oxidation of hydrocarbons by bacterial multicomponent monooxygenases (BMMs). ${ }^{2,3}$ Dioxygen activation and the conversion of $\mathrm{C}-\mathrm{H}$ to $\mathrm{C}-\mathrm{OH}$ bonds is accomplished at carboxylatebridged diiron units within the $\alpha$-sub-units of the hydroxylase components of these enzymes. 4 The active oxidant in some of these reactions has been trapped as a kinetically competent intermediate that forms upon reaction of the diiron(II) core with $\mathrm{O}_{2} \cdot{ }^{5}$ The binding of a small effector protein 6 to the hydroxylase facilitates this process. The evolutionarily related ${ }^{2}$ toluene/ $o$-xylene monooxygenase (ToMO) ${ }^{7}$ and soluble methane monooxygenase (sMMO) ${ }^{3}$ hydroxylases ToMOH and MMOH have nearly identical resting diiron(III) active-site structures, $4,8-10$ but ToMO cannot hydroxylate methane. Since crystallographic characterization of ToMOH has thus far been limited to a native, oxidized structure at $2.15 \AA$ resolution, ${ }^{8}$ and in order to expand our structural characterization and mechanistic understanding of the ToMO system, we have determined X-ray crystal structures of native ToMOH to $1.85 \AA$ resolution and of ToMOH reconstituted with $\mathrm{Mn}$ (II) as an analogue of the reduced, diiron(II) enzyme. ${ }^{11}$ The results, which provide insights that significantly advance our knowledge about the BMM family of enzymes, are that (i) formation of the dimetal(II) center is accompanied by a carboxylate shift and opening of a site for dioxygen binding and activation; (ii) a strategically placed, conserved asparagine residue in the dimetal(II) form of the enzyme undergoes a rotameric shift; and (iii) there exists a previously unidentified series of hydrophobic cavities analogous to those in $\mathrm{MMOH}$. A technical discovery of note in this study is the use of an annealing procedure during data collection that improved the resolution from 2.55 to $1.85 \AA$.

Since a suitable crystal of the diiron(II) form of ToMOH was unavailable, the dimanganese (II) derivative was prepared from the apo protein and $\mathrm{MnCl}_{2}$ and structurally characterized to 2.20 ̊. A 1.85 A resolution structure of native $\mathrm{ToMOH}_{\mathrm{Ox}}$ was determined using crystals grown in an identical manner. Detailed information about sample preparation, Mn reconstitution, crystal annealing, data collection, and structure solution and statistics is supplied as Supporting Information.

Although the overall topology and fold of the native protein are essentially identical to those of the previously published structure of $\mathrm{ToMOH}_{\mathrm{Ox}}, 8$ the active site differs (Figures 1, S1, and $\mathrm{S} 2)$. Foremost is the absence of a thioglycolate molecule bridging the two iron atoms, which

Supporting Information Available: Experimental details for sample preparation and crystallization, X-ray data collection, and structure solutions, as well as Tables S1 and S2 and Figures S1-S4 (PDF). This material is available free of charge via the Internet at http://pubs.acs.org. 
was not present in the current purification buffer (Supporting Information). Instead, the iron atoms are linked either by a hydroxide anion, as observed in MMOH structures, ${ }^{12}$ or by the terminal hydroxyl group of the polyethylene glycol (PEG) molecule found in the productbinding channel (vide infra). ${ }^{8}$ Additional differences between the diiron centers of the previously reported and the present higher resolution structure of $\mathrm{ToMOH}_{\mathrm{Ox}}$ are minor and are summarized in Table S2.

The structure of the $\mathrm{Mn}(\mathrm{II})-\mathrm{ToMOH}$ derivative is quite revealing. The carboxylate group of E231, which is analogous to E243 in $\mathrm{MMOH}$, undergoes a shift from its terminal, monodentate coordination mode in ToMOH to a bidentate-terminal, monodentate-bridging position (Figures 1 and S1). In conjunction with this carboxylate shift, the bridging hydroxide syn to the coordinated histidine ligands is displaced, while the one in the anti position becomes protonated and is semibridging, being $2.8 \AA$ from Mn1. This structure closely resembles the core geometry of both reduced and dimanganese(II)-reconstituted MMOH (Figure S3). ${ }^{11,12}$ The results are consistent with Mn being in the divalent state and strongly suggest that $\mathrm{ToMOH}_{\text {red }}$ will have the same configuration. As in the structures of $\mathrm{MMOH}_{\mathrm{Ox}}, \mathrm{MMOH}_{\text {red }}$, and $\mathrm{Mn}$ (II)-MMOH, an increase in the metal-metal distance from 3.1 to $3.3 \AA$ accompanies the carboxylate shift and oxidation state change.

The present $\mathrm{Mn}$ (II)-ToMOH results are nicely consistent with recently reported X-ray absorption spectroscopy (XAS) data for ToMOH red $^{13}$ This study indicated that the reduced active site is similar to that of $\mathrm{MMOH}_{\text {red }}$, both having iron coordination numbers between 5 and 6 and average Fe-Fe distances of 3.3-3.4 $\AA$. Moreover, the XAS spectra of the diferrous hydroxylases in the presence of their respective regulatory proteins, MMOB and ToMOD, closely resemble one another, as well as those for hydroxylases in the absence of the regulatory protein. Thus, it is probable that the active-site structures of ToMOH and MMOH immediately prior to $\mathrm{O}_{2}$ activation are very similar, based on the results reported here and the XAS data. Differences in reactivity, therefore, are likely to reflect the nature of the oxygenated intermediates and/or the surrounding protein scaffolds.

In this regard, it is noteworthy that accompanying changes in the oxidation state of the dimetallic center are conformational shifts analogous to those first discovered in a comparison of the $\mathrm{MMOH}_{\text {red }}$ and $\mathrm{MMOH}_{\mathrm{Ox}}$ structures. ${ }^{12}$ Precisely the same features can be identified by comparing the $\mathrm{Mn}(\mathrm{II})-\mathrm{ToMOH}$ and $1.85 \AA \mathrm{ToMOH}_{\mathrm{Ox}}$ structures. Conformational shifts in MMOH residues N214, E240, L244, and R245 in $\mathrm{MMOH}^{12}$ are reflected in the analogous N202, Q228, S232, and R233 amino acid side chains of ToMOH (Figure 2). These residues are located on surface-exposed regions of helices $\mathrm{E}$ and $\mathrm{F}$, adjacent to the diiron center, where the regulatory protein is postulated to bind in the MMO system $10,12,14$ and known to bind in the phenol hydroxylase (PH) system. 15 The shift in ToMOH N202 (Figures 1 and 2) is most significant because (i) this residue is strictly conserved among BMMs; (ii) the structures reported here reveal the dependence of the side-chain orientation on oxidation state in ToMOH and $\mathrm{MMOH}$; and (iii) the amide group in the analogous residue N204 in PHH is in hydrogen bond contact with the side-chain hydroxyl group of S72 (S111 in MMOB; S82 in T4MOD) of the $\mathrm{PH}$ regulatory component (PHM) in the PHH-PHM complex X-ray structure. 15

In the $\mathrm{ToMOH}_{\mathrm{ox}}$ structure, the $\mathrm{N} 202$ side chain points away from the diiron center and faces bulk solvent. In $\mathrm{Mn}$ (II)-ToMOH the side chain rotates inward, toward the active site. The resulting $80^{\circ}$ rotameric shift in the $\mathrm{N} 202$ side chain occurs exclusively about the $\mathrm{C}_{\alpha}-\mathrm{C}_{\beta}$ bond and is the largest among the ToMOH oxidation-state-dependent rotamer shifts thus far encountered. As with $\mathrm{MMOH}$, the asparagine side-chain rotamer shift affects the local electrostatics and topology of the protein surface between helices E and F directly above the active site. In the oxidized protein, the N202 carboxamide group protrudes from the protein surface and, in this manner, can serve as a hydrogen bond donor or acceptor. Upon reduction 
of the metal ions, this carboxamide group is directed into the "pore" region directly above the active site and is centered between helices $\mathrm{E}$ and $\mathrm{F}$.

Collectively, these observations reinforce prior claims that the conserved hydroxylase asparagine ${ }^{12}$ and regulatory component serine ${ }^{16}$ residues are key players in the interaction between the hydroxylase and regulatory protein components. They further suggest that regulatory component protein binding to the hydroxylase is related to the asparagine rotamer shift, which, in turn, depends on the diiron center oxidation state.

The morphology of the protein interior distinguishes $\mathrm{MMOH}$ and ToMOH. Xenon pressurization as well as halogenated substrate- and product-binding experiments with $\mathrm{MMOH}$ crystals reveal that both Xe and hydrocarbons can reside in a series of variably interconnected hydrophobic cavities in the R-subunit (Figure 3). ${ }^{14,17}$ The cavities, or pockets, trace a pathway from the active site to the protein surface and are postulated to be the means of substrate access to, or product egress from, the diiron center. Homologous pockets exist in ToMOH (Figure 3) but were not proposed as a link from the surface to the diiron center because of the occurrence of a 35-40 A channel that connects the active site to the protein exterior via a divergent pathway. The hydrophobic pockets that traverse the interior of the ToMOH R-subunit are the active-site pocket (cavity 1), an intermediate space located between helices B and D (cavity 2), and a solvent-exposed cavity found near the interface of the $\alpha$ and $\gamma$ subunits (cavity 3). Cavity 1 is structurally homologous to cavity 1 in $\mathrm{MMOH}$, and cavities 2 and 3 are homologous to cavity 2 and a combination of cavities 3 and 4 in $\mathrm{MMOH}$, respectively. It is possible that the hydrophobic pockets in ToMOH convey hydrocarbon access to the active site, but an alternative scenario is that, in $\mathrm{ToMOH}$, the cavities are a consequence of its evolutionary relationship to $\mathrm{MMOH}$. If the latter is the case, then the ToMOH channel may have been selected to tune its reactivity and favor hydroxylation of aromatic substrates.

In the $\mathrm{ToMOH}_{\mathrm{ox}}$ and $\mathrm{Mn}(\mathrm{II})-\mathrm{ToMOH}$ structures presented here, electron density in the channel has been modeled as a molecule of hexaethylene glycol (HEG), a component of PEG 400 in the crystallization buffer (Figure S4). Attempts to model water molecules into the density led to unreasonable interatomic distances. In both structures, HEG is partially disordered and exhibits high $B$-factors which generally increase with distance from the active site. Although our model indicates that the HEG chain ends at the channel fork, its degree of polymerization may be greater, with the remainder of the chain positionally disordered between the two channel branches. Moreover, for both structures, it was possible to model the end of the HEG molecule at the active-site metal ions either as a bridging alkoxide or simply in hydrogen bond contact with a bridging oxygen atom, presumably a hydroxide ion. These two models afforded similar refinement statistics and could not be distinguished. For presentation purposes, we have chosen the latter option, which can account for the channel electron density.

The present $\mathrm{Mn}(\mathrm{II})-\mathrm{ToMOH}$ and $\mathrm{ToMOH}_{\mathrm{OX}}$ structures contribute to the mounting structural evidence implicating BMM protein scaffolds as determinants of intersystem reaction diversity. The active sites of ToMOH and $\mathrm{MMOH}$ are highly homologous in both the reduced and the resting states. Control of conformational shifts by the redox state of the protein, first noted in $\mathrm{MMOH}$, is conserved in ToMOH. Experimentally, we show here that ToMOH is susceptible to iron extraction and $\mathrm{Mn}$ reconstitution by methods established in identical experiments with MMOH. ${ }^{11}$ Crystal an-nealing by careful cryostream control at SSRL induced a dramatic resolution increase in crystalline samples of $\mathrm{ToMOH}$, a procedure that should prove useful in future studies. The resulting resolution increase in the $\mathrm{ToMOH}_{\mathrm{ox}} \mathrm{X}$-ray structure has allowed us to improve our view of the protein interior and diiron center, leading to an enhanced description of the protein scaffold, active-site ligation, and core geometry. 


\section{Supplementary Material}

Refer to Web version on PubMed Central for supplementary material.

\section{Acknowledgment.}

This work was supported by a grant from the National Institutes of Health (GM32134 to S.J.L.). M.H.S. was supported under a NIH Biotechnology Training Grant. X-ray data were collected at the Stanford Synchrotron Radiation Laboratory (SSRL), funded by the Department of Energy (BES, BER) and the NIH (NCRR, NIGMS). Crystallographic coordinates have been deposited in the RCSB databank, accession numbers 2INC and 2IND. All figures were generated using PyMOL. ${ }^{18}$

\section{References}

(1). Tshuva EY, Lippard SJ. Chem. Rev 2004;104:987-1012. [PubMed: 14871147] and references cited therein

(2)(a). Notomista E, Lahm A, Di Donato A, Tramontano A. J. Mol. Evol 2003;56:435-445. [PubMed: 12664163]

(2)(b). Leahy JG, Batchelor PJ, Morcomb SM. FEMS Microbiol. Rev 2003;27:449-479. [PubMed: 14550940]

(3). Merkx M, Kopp DA, Sazinsky MH, Blazyk JL, Müller J, Lippard SJ. Angew. Chem., Int. Ed 2001;40:2782-2807.

(4). Sazinsky MH, Lippard SJ. Acc. Chem. Res 2006;39:558-566. [PubMed: 16906752]

(5)(a). Murray LJ, García-Serres R, Naik S, Huynh BH, Lippard SJ. J. Am. Chem. Soc 2006;128:74587459. [PubMed: 16756297]

(5)(b). Beauvais LG, Lippard SJ. J. Am. Chem. Soc 2005;127:7370-7378. [PubMed: 15898785]

(5)(c). Brazeau BJ, Lipscomb JD. Biochemistry 2000;39:13503-13515. [PubMed: 11063587]

(5)(d). Valentine AM, Stahl SS, Lippard SJ. J. Am. Chem. Soc 1999;121:3876-3887.

(5)(e). Shu L, Nesheim JC, Kauffmann K, Münck E, Lipscomb JD, Que L Jr. Science 1997;275:515518. [PubMed: 8999792]

(5)(f). Liu KE, Wang D, Huynh BH, Edmondson DE, Salifoglou A, Lippard SJ. J. Am. Chem. Soc 1994;116:7465-7466.

(5)(g). Lee S-K, Nesheim JC, Lipscomb JD. J. Biol. Chem 1993;268:21569-21577. [PubMed: 8408008]

(6)(a). Zhang J, Wallar BJ, Popescu CV, Renner DB, Thomas DD, Lipscomb JD. Biochemistry 2006;45:2913-2926. [PubMed: 16503646] and references cited therein

(6)(b). Lountos GT, Mitchell KH, Studts JM, Fox BG, Orville AM. Biochemistry 2005;44:7131-7142. [PubMed: 15882052]

(6)(c). Scognamiglio R, Notomista E, Barbieri P, Pucci P, Dal Piaz F, Tramontano A, Di Donato A. Protein Sci 2001;10:482-490. [PubMed: 11344317]

(6)(d). Walters KJ, Gassner GT, Lippard SJ, Wagner G. Proc. Natl. Acad. Sci. U.S.A 1999;96:78777882. [PubMed: 10393915]

(6)(e). Liu Y, Nesheim JC, Lee S-K, Lipscomb JD. J. Biol. Chem 1995;270:24662-24665. [PubMed: 7559577]

(7). Cafaro V, Izzo V, Scognamiglio R, Notomista E, Capasso P, Casbarra A, Pucci P, Di Donato A. Appl. Environ. Microbiol 2004;70:2211-2219. [PubMed: 15066815] and references cited therein

(8). Sazinsky MH, Bard J, Di Donato A, Lippard SJ. J. Biol. Chem 2004;279:30600-30610. [PubMed: 15096510]

(9)(a). Rosenzweig AC, Brandstetter H, Whittington DA, Nordlund P, Lippard SJ, Frederick CA. Proteins 1997;29:141-152. [PubMed: 9329079]

(9)(b). Elango N, Radhakrishnan R, Froland WA, Wallar BJ, Earhart CA, Lipscomb JD, Ohlendorf DH. Protein Sci 1997;6:556-568. [PubMed: 9070438]

(9)(c). Rosenzweig AC, Nordlund P, Takahara PM, Frederick CA, Lippard SJ. Chem. Biol 1995;2:409_ 418. 
(10). Rosenzweig AC, Frederick CA, Lippard SJ, Nordlund P. Nature 1993;366:537-543. [PubMed: 8255292]

(11). Sazinsky MH, Merkx M, Cadieux E, Tang S, Lippard SJ. Biochemistry 2004;43:16263-16276. [PubMed: 15610020]

(12). Whittington DA, Lippard SJ. J. Am. Chem. Soc 2001;123:827-838. [PubMed: 11456616]

(13). Rudd DJ, Sazinsky MH, Lippard SJ, Hedman B, Hodgson KO. Inorg. Chem 2005;44:4546-4554. [PubMed: 15962961]

(14). Sazinsky MH, Lippard SJ. J. Am. Chem. Soc 2005;127:5814-5825. [PubMed: 15839679]

(15). Sazinsky MH, Dunten PW, McCormick MS, Di Donato A, Lippard SJ. Biochemistry. 2006in press

(16). Zheng H, Lipscomb JD. Biochemistry 2006;45:1685-1692. [PubMed: 16460015]

(17). Whittington DA, Rosenzweig AC, Frederick CA, Lippard SJ. Biochemistry 2001;40:3476-3482. [PubMed: 11297413]

(18). DeLano, WL. The PyMOL Molecular Graphics System. DeLano Scientific; San Carlos, CA: 2002. JA064837R 

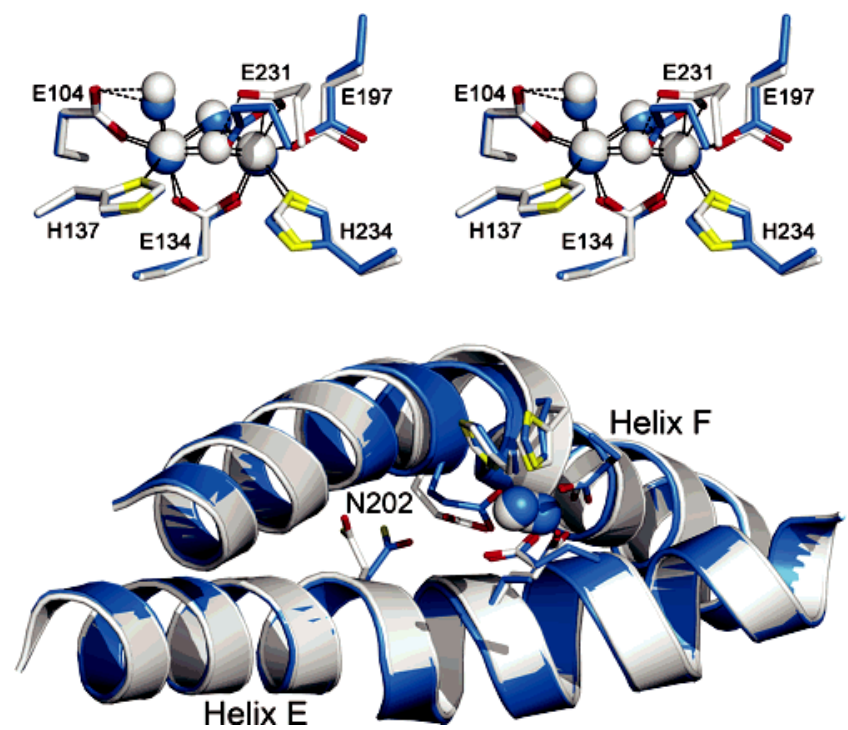

Figure 1.

Overlaid 1.85 ̊ ToMOHox (white) and 2.20 ̊ Mn(II)-ToMOH (blue) structures. (Top) Stereoview of the active sites. (Bottom) Helices E and F, active-site ligands and metals, and N202. Fe(III) ions, Mn(II) ions, and oxygen atom species are represented as white or blue spheres. Side-chain ligands are represented as sticks in white or blue (carbon), red (oxygen), and yellow (nitrogen). 

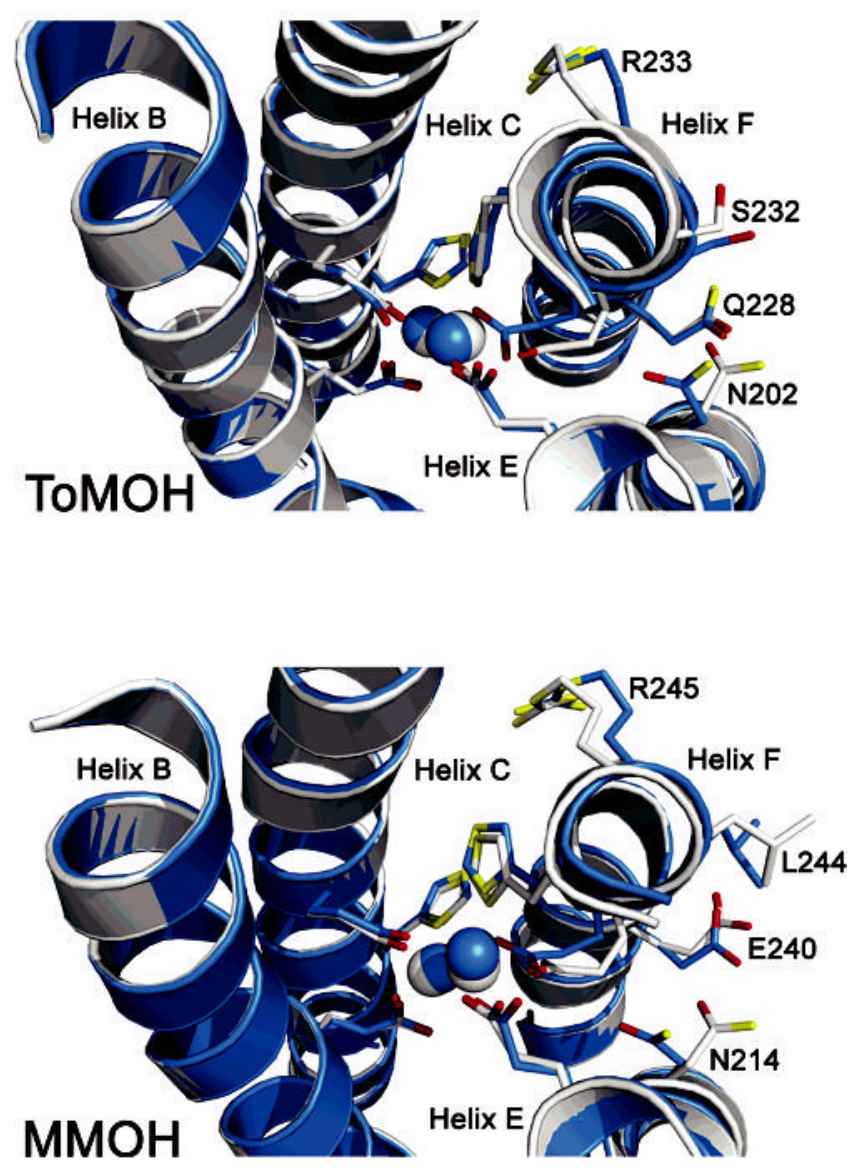

Figure 2.

Metal center oxidation-state-dependent conformational shifts in ToMOH and MMOH. (Top) $1.85 \AA \mathrm{ToMOH}_{\mathrm{OX}}$ (white) and $2.20 \AA \mathrm{Mn}$ (II)-ToMOH (blue). (Bottom) $\mathrm{MMOH}_{\mathrm{OX}}$ (white; PDB code $1 \mathrm{MTY}$ ) and $\mathrm{MMOH}_{\text {red }}$ (blue; PDB code 1FYZ). The metal-binding four-helix bundle, active-site ligands and ions, and select side chains are shown as overlaid structures. Metal ions and helix backbones are represented as spheres and cartoons, respectively. Amino acid side chains are shown as sticks in blue/white (carbon), red (oxygen), and yellow (nitrogen). 

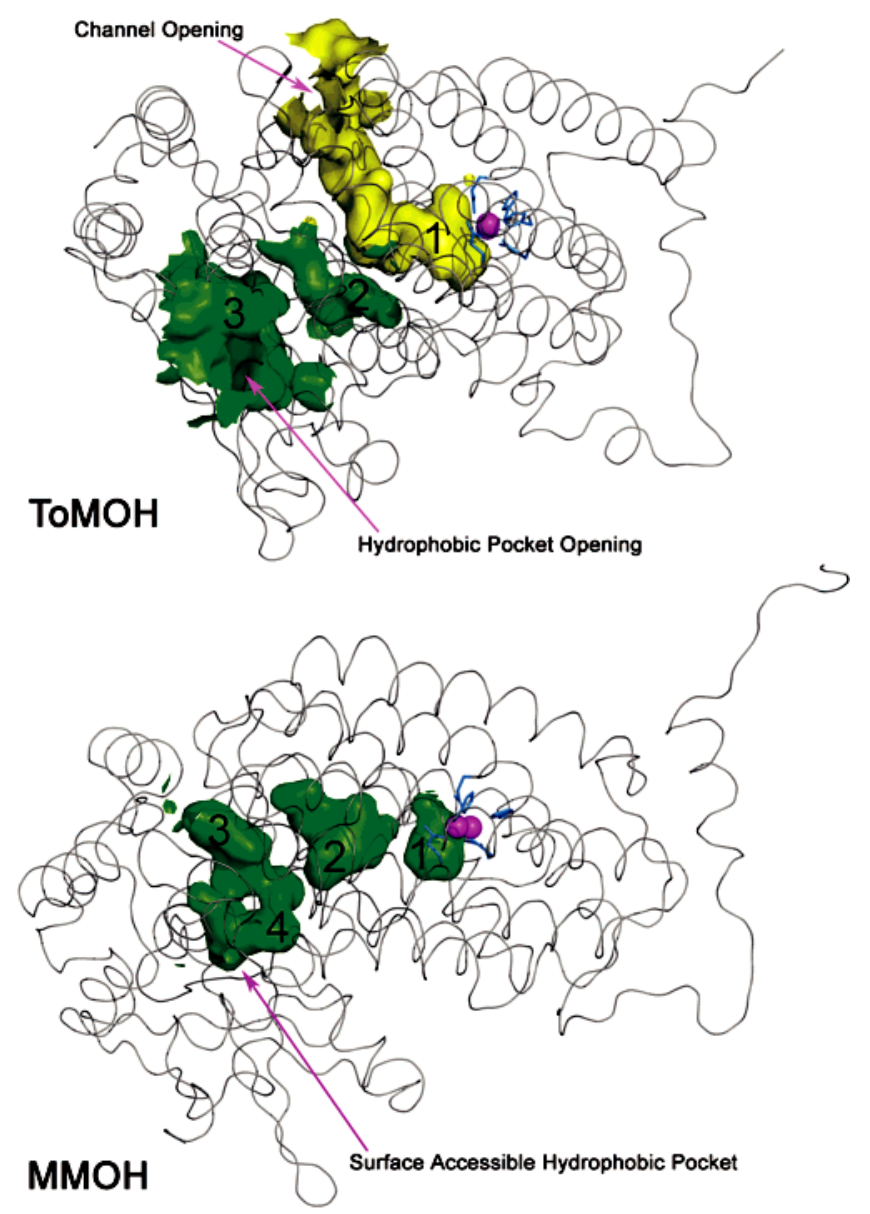

Figure 3.

Interior surface renderings of ToMOH (top) and $\mathrm{MMOH}$ (bottom) R-subunits. ToMOH channel van der Waals surface is shown in yellow; hydrophobic pockets in ToMOH and MMOH are shown in green. Protein backbones $\left(\mathrm{C}_{\alpha}\right.$ trace $)$ are represented as gray ribbons, active-site iron atoms as magenta spheres, and side-chain ligands as blue sticks. 